\title{
ON THE CARDINALITY OF COUNTABLE DENSE HOMOGENEOUS SPACES
}

\author{
A. V. ARHANGEL'SKII AND J. VAN MILL \\ (Communicated by Alexander N. Dranishnikov)
}

\begin{abstract}
We prove that a countable dense homogeneous space has size at most continuum. If moreover it is compact, then it is first-countable under the Continuum Hypothesis. We also construct under the Continuum Hypothesis an example of a hereditarily separable, hereditarily Lindelöf, countable dense homogeneous compact space of uncountable weight.
\end{abstract}

\section{INTRODUCTION}

All spaces under discussion are Hausdorff.

A separable space $X$ is countable dense homogeneous (abbreviated: $\mathrm{CDH}$ ) if given any two countable dense subsets $D$ and $E$ of $X$, there is a(n) (auto)homeomorphism $f: X \rightarrow X$ such that $f(D)=E$. This notion is of interest only if $X$ is separable, so we include separability in its definition.

The first result in this area is due to Cantor [5], who showed by his now famous 'back-and-forth' method that the reals $\mathbb{R}$ are $\mathrm{CDH}$. Fréchet and Brouwer, independently, proved that the same is true for the $n$-dimensional Euclidean space $\mathbb{R}^{n}$. Fort [10] proved that the Hilbert cube is also $\mathrm{CDH}$. There are many other CDHspaces. Bessaga and Pełczyński 3] proved that every strongly locally homogeneous Polish space is $\mathrm{CDH}$. Hence spaces such as the irrational numbers and separable Hilbert space are CDH. Farah, Hrušák and Martínez Ranero [8] proved that there is a subspace of $\mathbb{R}$ of size $\aleph_{1}$ that is $\mathrm{CDH}$. For more examples, results and references, we refer the reader to the survey [1].

The topological sum of the 1-spheres $\mathbb{S}^{1}$ and $\mathbb{S}^{2}$ is an example of a $\mathrm{CDH}$-space that is not homogeneous. Bennett 2 proved in 1972 that a connected first-countable $\mathrm{CDH}$-space is homogeneous. (The converse is not true, and the assumption on firstcountability is superfluous; see 9].) Hence for connected spaces, countable dense homogeneity can be thought of as a strong form of homogeneity.

Countable dense homogeneity was studied mainly within the class of separable metrizable spaces. Actually, except for some general homogeneity results, there are very few results known for countable dense homogeneous spaces that are Tychonoff but not metrizable. Steprāns and Zhou [15] proved that every separable manifold of weight less than $\mathfrak{b}$ is $\mathrm{CDH}$ and also that the Cantor cube $2^{\omega_{1}}$ is $\mathrm{CDH}$ under $\mathrm{MA}+\neg \mathrm{CH}$. (For information on small uncountable cardinals, see [7].) But to obtain

Received by the editors December 16, 2011 and, in revised form, January 10, 2012.

2010 Mathematics Subject Classification. Primary 54A25, 54D65, 54H11.

Key words and phrases. Countable dense homogeneous, cardinality, Continuum Hypothesis. 
interesting examples from these results, one needs additional set theoretic assumptions. Much to our surprise, it seems unknown whether there is a $\mathrm{CDH}$-compactum of uncountable weight in ZFC.

We prove that every $\mathrm{CDH}$-space is of size at most $2^{\omega}$ and that a regular $\mathrm{CDH}$ space is first-countable provided it is first-countable at a dense set of points. As a corollary, we obtain that under $2^{\omega}<2^{\omega_{1}}$, every $\mathrm{CDH}$-compactum is first-countable. The result of Steprāns and Zhou [15] that the Cantor cube $2^{\omega_{1}}$ is CDH under $\mathrm{MA}+\neg \mathrm{CH}$ shows that this is not a ZFC-result. We also present under the Continuum Hypothesis, abbreviated $\mathrm{CH}$, an example of a nonmetrizable compact and zero-dimensional CDH-space; our space is even hereditarily separable and hereditarily Lindelöf. Our construction was inspired by Kunen's well-known example of a compact L-space under $\mathrm{CH}, 12$. A natural candidate for a $\mathrm{CDH}$-compactum that is not metrizable is the Alexandroff-Urysohn double arrow space. We show, however, that this space is not $\mathrm{CDH}$.

The following fundamental problem remains open.

Question 1.1. Does there exist in ZFC a compact CDH-space that is not metrizable?

\section{ON THE CARDinAlity OF CDH-SPACES}

Let us call a space $X$ a $c_{1}$-space if the following condition $\left(c_{1}\right)$ is satisfied: $\left(c_{1}\right)$ $X$ is separable, and every two countable dense subspaces of $X$ are homeomorphic. Let us call a space $X$ a $c_{2}$-space if the following condition $\left(c_{2}\right)$ is satisfied: $\left(c_{2}\right) X$ is separable, and every countable dense subspace of $X$ is homogeneous.

Sierpiński [14] proved that the space of rational numbers $\mathbb{Q}$ is topologically the unique nonempty regular countable and first-countable space without isolated points. Hence every compact space with countable weight and without isolated points is a $c_{1}$-space. This means that there are many compact spaces that are $c_{1}$ but not $\mathrm{CDH}$, for example the product of the Cantor set and the circle, which is even a topological group. There does not exist a compact zero-dimensional space of countable weight which is $c_{1}$ but not $\mathrm{CDH}$. This follows easily from the topological characterization of the Cantor set due to Brouwer [4].

Theorem 2.1. If $X$ is a $c_{1}$-space, then $|X| \leq 2^{\omega}$.

Proof. Fix a countable dense subspace $M$ of $X$, and put $Y=X \backslash M$. For each $y \in Y$, put $Z_{y}=M \cup\{y\}$. The subspaces $M$ and $Z_{y}$ of $X$ are homeomorphic, by our assumption.

Therefore, for each $y \in Y$, we can fix a homeomorphism $f_{y}$ of $M$ onto $Z_{y}$.

Let $x_{y}$ be the preimage of $y$ under $f_{y}$. Put $M_{y}=M \backslash\left\{x_{y}\right\}$, and denote by $g_{y}$ the restriction of $f_{y}$ to $M_{y}$. Observe that $x_{y}$ is not isolated in $M$. Hence, $M_{y}$ is dense in $M$.

Assume now that the cardinality of $Y$ exceeds $2^{\omega}$. Then there are two distinct points $p$ and $q$ in $Y$ such that $x_{p}=x_{q}, g_{p}=g_{q}$, and $M_{p}=M_{q}$ (since the cardinality of the set of all possible mappings of arbitrary subsets of $M$ to $M$ doesn't exceed $\left.2^{\omega}\right)$.

However, this is impossible, since it says that the same continuous mapping $h=g_{p}=g_{q}$ of the dense subspace $H=M_{p}=M_{q}$ of the space $M$ to the Hausdorff space $X$ can be extended to a continuous mapping of the space $M$ into $X$ in two different ways ( $f_{p}$ and $f_{q}$ are the two distinct extensions). 
Theorem 2.2. Suppose that $X$ is a $c_{1}$-space which contains a dense first-countable subspace $E$. Moreover, assume that $X$ is regular. Then $X$ is first-countable.

Proof. Since $X$ is a regular $T_{1}$-space, it follows that for every dense subspace $S$ of $X$ and $p \in S$ we have $\chi(p, S)=\chi(p, X)$, [11, 2.1]. This will be used several times without explicit reference in this proof.

Hence, we may assume that $E$ consists of all points of first-countability of $X$. We will show that $E=X$.

Claim 1. $E$ is open in $X$.

Assume that $E$ is not open in $X$. Let $t \in E$ be in the closure of $X \backslash E$. Since $X$ is first-countable at $t$, one can find a countable dense set $D$ in $X$ such that $t$ is in the closure of $C=D \backslash E$ (let $D$ be the union of a sequence in $X \backslash E$ converging to $t$ and an arbitrary countable dense set). Let $S=D \cap E$ and put $D^{\prime}=(S \backslash \bar{C}) \cup C$. It is straightforward to check that $D^{\prime}$ is a countable subset of $D$ dense in $X$. Put $D^{\prime \prime}=D^{\prime} \cup\{t\}$. Since $X$ is a $c_{1}$-space, there exists a homeomorphism $h$ of $D^{\prime \prime}$ onto $D^{\prime}$. Since $t \in E$ and $D^{\prime}$ is dense in $X$, the space $D^{\prime}$ is first countable at $h(t)$. Hence $h(t) \in E$, and so $h(t) \notin \bar{C}$. Since, obviously, $h(C) \subseteq C$, it follows that $t$ is not in the closure of $C$. This is a contradiction.

It follows from the claim that the set $S=D \cap E$ is dense in $X$. Thus, $S$ is a countable first-countable dense subspace of $X$. Now take any $t \in X$. Then $S \cup\{t\}$ is homeomorphic to $S$. It consequently follows that $X$ is first-countable at $t$. Hence, $X=E$.

Remark 2.3. The same result holds for $c_{2}$-spaces, with an almost identical proof.

Corollary 2.4. If $X_{i}$ is regular and has a dense first-countable subspace for each $i \in \omega$ and if $\prod_{i \in \omega} X_{i}$ is $c_{1}$, then each $X_{i}$ is first-countable.

Corollary $2.5\left(2^{\omega}<2^{\omega_{1}}\right)$. Every $c_{1}$-compactum is first-countable.

Proof. Let $X$ be a $c_{1}$-compactum. Then $|X| \leq 2^{\omega}$ by Theorem 2.1. It consequently follows from $2^{\omega}<2^{\omega_{1}}$ that $X$ is first-countable at a dense set of points, [11, 2.22], and so we are done by Theorem 2.2

Remark 2.6. By Steprāns and Zhou [15, the Cantor cube $2^{\omega_{1}}$ is a CDH-space under $\mathrm{MA}_{\aleph_{1}}$. Hence Corollary 2.5 cannot be proved in ZFC, but it can be considerably generalized, with essentially the same proof, as follows. Recall that a space $X$ is of pointwise countable type if every $x \in X$ is contained in a compact subspace $F$ of $X$ with a countable base of open neighbourhoods in $X$.

Corollary $2.7\left(2^{\omega}<2^{\omega_{1}}\right)$. Every $c_{1}$-space of pointwise countable type is firstcountable.

This statement covers locally compact $c_{1}$-spaces, Čech-complete $c_{1}$-spaces, and even $c_{1}$-spaces that are $p$-spaces.

\section{The Double ARrow SPACE}

We now address the natural problem of whether the Alexandroff-Urysohn double arrow space is $\mathrm{CDH}$.

Consider the real line $\mathbb{R}$. We split each $t \in \mathbb{R}$ into two points, $t^{-}$and $t^{+}$. Order the set $\mathbb{A}=\left\{t^{-}, t^{+}: t \in \mathbb{R}\right\}$ in the natural way so that $t^{-}$always precedes $t^{+}$. If 
we compactify $\mathbb{A}$ with $-\infty$ and $+\infty$, then we obtain the Alexandroff double arrow space $\hat{\mathbb{A}}$.

If $A \subseteq \mathbb{R}$, then $A^{-}$denotes $\left\{a^{-}: a \in A\right\}$. Similarly for $A^{+}$. Observe that $\mathbb{R}^{+}$ and $\mathbb{R}^{-}$are both copies of the Sorgenfrey line.

The following result is due to van Douwen [6, p. 160]. He did not present its proof, but instead hinted at a similar result for which the proof is given in the paper. For the convenience of the reader, we present all the details.

Lemma 3.1. Let $A$ be second category in $\mathbb{R}^{+}$, and let $f: A \rightarrow \mathbb{R}^{+}$be an embedding. Then there are $s, t \in \mathbb{R}^{+}$such that $s<t,(s, t) \cap A$ is second category in $\mathbb{R}^{+}$and $f \uparrow(s, t) \cap A$ is strictly increasing.

Proof. There is $n \in \mathbb{N}$ such that the set

$$
A_{0}=\left\{a \in A:[a, a+1 / n) \cap A \subseteq f^{-1}([f(a), \rightarrow))\right\}
$$

is second category in $\mathbb{R}^{+}$. Since $\mathbb{R}^{+}$is hereditarily Lindelöf, we may assume that $A_{0}$ is everywhere second category in $\mathbb{R}^{+}$. Now pick $s, t \in \mathbb{R}^{+}$such that $s<t$, $t-s<1 / n$, and $A_{0} \cap(s, t)$ is dense in $(s, t)$. Pick $x, y \in(s, t) \cap A$ such that $x<y$ and $f(y)<f(x)$. There are sequences $\left(a_{n}\right)_{n}$ and $\left(b_{n}\right)_{n}$ in $A_{0} \cap(s, t)$ such that $a_{n} \searrow x$ and $b_{n} \searrow y$. There consequently exists $n$ such that $x<a_{n}<y<b_{n}$ and $f(y)<f\left(b_{n}\right)<f(x)<f\left(a_{n}\right)$. But this is impossible since $b_{n} \in\left[a_{n}, a_{n}+1 / n\right)$; hence $f\left(b_{n}\right) \geq f\left(a_{n}\right)$.

Theorem 3.2. The Alexandroff-Urysohn double arrow space is not $\mathrm{CDH}$ (but it is clearly $\left.c_{1}\right)$.

Proof. We represent the double arrow space by $\{-\infty\} \cup \mathbb{A} \cup\{+\infty\}$. Split $\mathbb{Q}$ into two dense sets $\mathbb{Q}_{1}$ and $\mathbb{Q}_{2}$ such that $-\mathbb{Q}_{1}=\mathbb{Q}_{2}$. The function $i: \mathbb{A} \rightarrow \mathbb{A}$ defined by

$$
i\left(t^{-}\right)=-t^{+}, \quad i\left(t^{+}\right)=-t^{-}
$$

is an involution, and $i\left(\mathbb{Q}_{1}^{+}\right)=\mathbb{Q}_{2}^{-}$.

Assume that there is a homeomorphism $f: \hat{\mathbb{A}} \rightarrow \widehat{\mathbb{A}}$ such that

$$
f\left(\mathbb{Q}^{-} \cup \mathbb{Q}^{+}\right)=\mathbb{Q}_{1}^{+} \cup \mathbb{Q}_{2}^{-} .
$$

The space $X=\mathbb{R}^{+} \backslash f^{-1}(\{-\infty,+\infty\})$ is a Baire space; hence either the set $\{x \in$ $\left.X: f(x) \in \mathbb{R}^{+}\right\}$or the set $\left\{x \in X: f(x) \in \mathbb{R}^{-}\right\}$is second category in $\mathbb{R}^{+}$. In the second case we consider the composition $i \circ f$. This shows that we may assume without loss of generality that the set

$$
S=\left\{t^{+} \in \mathbb{R}^{+}: f\left(t^{+}\right) \in \mathbb{R}^{+}\right\}
$$

is second category in $\mathbb{R}^{+}$. Hence we can find by Lemma 3.1 elements $s, t \in \mathbb{R}^{+}$ such that $s<t$ and $f\left\lceil(s, t) \cap \mathbb{R}^{+}\right.$is strictly increasing. Since $(s, t) \cap \mathbb{R}^{+}$is dense in $(s, t)$, this means that $f\lceil(s, t)$ is strictly increasing. Pick a nontrivial interval of the form $\left[a^{+}, b^{-}\right]$which is contained in $(s, t)$. Then $f\left(\left[a^{+}, b^{-}\right]\right)$is a compact and clopen subset of $\mathbb{A}$. Hence there is a sequence

$$
s_{1}^{+}<t_{1}^{-}<s_{2}^{+}<t_{2}^{-}<\cdots<s_{n}^{+}<t_{n}^{-}
$$

in $\mathbb{A}$ such that $f\left(\left[a^{+}, b^{-}\right]\right)=\left[s_{1}^{+}, t_{1}^{-}\right] \cup \cdots \cup\left[s_{n}^{+}, t_{n}^{-}\right]$. Pick an arbitrary rational number $q$ such that $a<q<b$. Observe that the points $f\left(q^{-}\right)$and $f\left(q^{+}\right)$form a jump in $f\left(\left[a^{+}, b^{-}\right]\right)$. Also observe that $f\left(\mathbb{Q}^{-} \cup \mathbb{Q}^{+}\right)=\mathbb{Q}_{1}^{+} \cup \mathbb{Q}_{2}^{-}$does not contain any pair of the form $x^{-}, x^{+}$for $x \in \mathbb{Q}$. Hence $f\left(q^{-}\right)$and $f\left(q^{+}\right)$have to be of the 
form $t_{1}^{-}, s_{2}^{+}$, etc. But there are only finitely many such jumps and infinitely many rational numbers between $a$ and $b$. This is a contradiction.

Question 3.3. Let $X$ be the product of the double arrow space and the Cantor set. Is $X \mathrm{CDH}$ ?

\section{A compact first-Countable CDH-Space of uncountable Weight}

We will now show that, under $\mathrm{CH}$, there is a compact $\mathrm{CDH}$-space $X$ of uncountable weight. In fact, $X$ is both hereditarily separable and hereditarily Lindelöf.

A subset $A$ of a space is called regular open if it is the interior of its own closure. As usual, $\mathrm{RO}(X)$ denotes the collection of all regular open subsets of $X$. It is well known that $\mathrm{RO}(X)$ is a Boolean algebra under natural operations. For details, see e.g. 13 .

For a space $X$, we let $\mathscr{H}(X)$ denote its group of homeomorphisms.

Let $K$ denote the standard Cantor set in $\mathbb{I}=[0,1]$.

Lemma 4.1. Let $G$ be a countable subgroup of $\mathscr{H}(K)$, and assume that some dense first category subset $D$ of $K$ is invariant under every element in $G$. Then there is a Cantor set $K_{1}$ and a continuous surjection $\pi: K_{1} \rightarrow K$ having the following properties:

(1) for every $d \in D, \pi^{-1}(d)$ is a single point,

(2) $\pi^{-1}(D)$ is dense in $K_{1}$ (hence $\pi$ is irreducible),

(3) for some $x \in K \backslash D, \pi^{-1}(x)$ contains at least two elements,

(4) there is an (algebraic) embedding $i: G \rightarrow \mathscr{H}\left(K_{1}\right)$ such that $g \circ \pi=\pi \circ i(g)$ for every $g \in G$.

Proof. Let $\mathscr{C}$ denote the subalgebra of $\mathrm{RO}(K)$ consisting of all clopen subsets of $K$. Moreover, for some $x \in K \backslash D$, pick two disjoint regular open subsets $U$ and $V$ in $K$ such that $\{x\}=\bar{U} \cap \bar{V}$. There is a countable subalgebra $\mathscr{E}$ of $\mathrm{RO}(K)$ which contains $\mathscr{C}$ as well as $U$ and $V$ and which is invariant under every element of $G$. The Stone space $K_{1}$ of $\mathscr{E}$ has all desired properties. For (4), simply observe that for every $g \in G$ there is a unique element $g^{*} \in \mathscr{H}\left(K_{1}\right)$ such that $g \circ \pi=\pi \circ g^{*}$ and that the uniqueness of $g^{*}$ implies that the assignment $g \mapsto g^{*}$ is an algebraic embedding.

Assume $\mathrm{CH}$ from now on. Put $X_{0}=K$, and let $D_{0}$ be a fixed countable dense subset of $X_{0}$, and let $G_{0}$ be the subgroup of $\mathscr{H}\left(X_{0}\right)$ consisting only of the neutral element. We will inductively construct spaces $X_{\alpha}$ for $\alpha \leq \omega_{1}$. The example $X$ we are looking for will be $X_{\omega_{1}}$. Each $X_{\alpha}$ will be a closed subspace of $K^{\alpha}$. If $\alpha \leq \beta$, let $\pi_{\alpha}^{\beta}: K^{\beta} \rightarrow K^{\alpha}$ be the projection. We shall organize things so that:

(A) If $\alpha \leq \beta$, then $\pi_{\alpha}^{\beta}\left(X_{\beta}\right)=X_{\alpha}$.

The restriction of the projection $\pi_{\alpha}^{\beta}$ to $X_{\beta}$ will be denoted by $p_{\alpha}^{\beta}$. The construction is determined at limit ordinals $\gamma \leq \omega_{1}$. For such $\gamma$ set

$$
X_{\gamma}=\left\{x \in K^{\gamma}:(\forall \alpha<\gamma)\left(\pi_{\alpha}^{\gamma}(x) \in X_{\alpha}\right)\right\} .
$$

Hence $X_{\gamma}$ is the inverse limit of the sequence $\left\{X_{\alpha}: \alpha<\gamma\right\}$.

We will also construct for $\beta<\omega_{1}$ a dense first category $F_{\sigma}$-subset $D_{\beta}$ of $X_{\beta}$ and a countable subgroup $G_{\beta}$ of $\mathscr{H}\left(X_{\beta}\right)$ such that the following conditions are satisfied:

(B) for every $g \in G_{\beta}$ we have that $g\left(D_{\beta}\right)=D_{\beta}$,

(C) for $\alpha<\beta$ and $d \in D_{\alpha},\left(p_{\alpha}^{\beta}\right)^{-1}(d)$ is a single point that belongs to $D_{\beta}$, 
(D) for $\alpha<\beta,\left\{\left(\pi_{\alpha}^{\beta}\right)^{-1}(d): d \in D_{\alpha}\right\}$ is dense in $X_{\beta}$,

(E) for $\alpha<\beta$ and $g \in G_{\alpha}$, there exists an element $h \in G_{\beta}$ such that $g \circ p_{\alpha}^{\beta}=$ $p_{\alpha}^{\beta} \circ h$.

As we already said, at limit $\gamma$ the space $X_{\gamma}$ is determined. Moreover, by (C) and the fact that all maps involved are irreducible,

$$
D_{\gamma}=\bigcup_{\alpha<\gamma}\left(p_{\alpha}^{\gamma}\right)^{-1}\left(D_{\alpha}\right)
$$

is a dense first category $F_{\sigma}$-subset of $X_{\gamma}$. Also, (D) and (E) and the fact that $X_{\gamma}$ is the inverse limit of the sequence $\left\{X_{\alpha}: \alpha<\gamma\right\}$ imply that for every $\alpha<\gamma$ and $g \in G_{\alpha}$ there is a unique homeomorphism $h \in \mathscr{H}_{\gamma}$ such that $g \circ p_{\alpha}^{\gamma}=p_{\alpha}^{\gamma} \circ h$. Also, $h$ clearly has the property that $h\left(D_{\gamma}\right)=D_{\gamma}$. The collection of these $h$ 's is a countable subgroup $H$ of $\mathscr{H}\left(X_{\gamma}\right)$. Put $G_{\gamma}=H$.

We now deal with the successor steps. Fix an enumeration $\left\{E_{\xi}^{\alpha}: \xi<\omega_{1}\right\}$ of all countable subsets of $K^{\alpha}$ for $\alpha<\omega_{1}$. Moreover, fix an enumeration $\left\{F_{\xi}^{\alpha}: \xi<\omega_{1}\right\}$ of all closed subsets of $K^{\alpha}$ for $\alpha<\omega_{1}$. Finally, fix a map $g$ from $\omega_{1}$ onto $\omega_{1} \times \omega_{1}$ such that $g(\beta)=\langle\alpha, \xi\rangle$ implies $\alpha \leq \beta$. We demand:

(F) For each $\beta<\omega_{1}$, if $g(\beta)=\langle\alpha, \xi\rangle$ and $E_{\xi}^{\alpha}$ is dense in $X_{\alpha}$ and contained in $D_{\alpha}$, then there is an element $g \in G_{\beta+1}$ such that

$$
g\left(\left(p_{0}^{\beta+1}\right)^{-1}\left(D_{0}\right)\right)=\left(p_{\alpha}^{\beta+1}\right)^{-1}\left(E_{\xi}^{\alpha}\right) .
$$

(G) For each $\beta<\omega_{1}$, if $F_{\xi}^{\alpha}$ is a nowhere dense closed subset of $X_{\alpha}$, then $\left(p_{0}^{\beta+1}\right)^{-1}\left(F_{\xi}^{\alpha}\right)$ is contained in $D_{\beta+1}$.

(H) For each $\beta<\omega_{1}$ there exists an element $x \in X_{\beta}$ such that $\left(p_{\beta}^{\beta+1}\right)^{-1}(x)$ contains more than one point.

This is easy to arrange. Assume that we are in the case where for $(F)$ and $(G)$ there is work to be done. First observe that $X_{\beta}$ is a Cantor set and hence is CDH. Hence there is a homeomorphism $h: X_{\beta} \rightarrow X_{\beta}$ such that

$$
h\left(\left(p_{0}^{\beta}\right)^{-1}\left(D_{0}\right)\right)=\left(p_{\alpha}^{\beta}\right)^{-1}\left(E_{\xi}^{\alpha}\right) .
$$

Now let $G$ be the subgroup of $\mathscr{H}\left(X_{\beta}\right)$ generated by $G_{\beta} \cup\{h\}$. Put

$$
D=\bigcup_{h \in G} h\left(D_{\beta} \cup\left(p_{\alpha}^{\beta}\right)^{-1}\left(F_{\xi}^{\alpha}\right)\right) .
$$

Observe that the irreducibility of all maps involved implies that $D$ is a dense first category $F_{\sigma}$-subset of $X_{\beta}$, and so we can apply Lemma 4.1 with $K=K_{\beta}$ and $G$ and $D$ as stipulated, thus obtaining $K_{1}, i$ and $\pi$. We may assume that $K_{1}$ is a subspace of $K$. Now put

$$
X_{\beta+1}=\left\{\langle\pi(x), x\rangle: x \in K_{1}\right\} \subseteq K^{\beta} \times K .
$$

Here we make the obvious identification of $K^{\beta+1}$ with $K^{\beta} \times K$. If we identify $K_{1}$ and $X_{\beta+1}$, then it is clear from Lemma 4.1 how to define $G_{\beta+1}$ and $D_{\beta+1}$. Indeed, $G_{\beta+1}=i\left(G_{\beta}\right)$ and $D_{\beta+1}=\pi^{-1}(D)$.

This completes the construction. It remains to verify that $X$ has all desired properties.

It is clear that $X$ is separable, since the set $\left(p_{0}^{\omega_{1}}\right)^{-1}\left(D_{0}\right)$ is dense in $X$. Moreover, $X$ has uncountable weight since if it had countable weight, then there would be an index $\beta<\omega_{1}$ such that $p_{\beta}^{\omega_{1}}$ is a homeomorphism. But this is not the case by $(\mathrm{H})$. 
An almost Luzin space (Kunen [12]) is one in which every nowhere dense set is second countable in its relative topology. It was shown in [12, Lemma 1.2] that an almost Luzin space is hereditarily Lindelöf.

Lemma 4.2. For each nowhere dense closed subset $A$ of $X$, there exists $\beta<\omega_{1}$ such that $p_{\beta}^{\omega_{1}}(A) \subseteq D_{\beta}$; hence $\left(p_{\beta}^{\omega_{1}}\right)^{-1}\left(p_{\beta}^{\omega_{1}}(A)\right)=A$, and $p_{\beta}^{\omega_{1}}\left\lceil A: A \rightarrow p_{\beta}^{\omega_{1}}(A)\right.$ is a homeomorphism. Hence $X$ is almost Luzin.

Proof. Since $X$ is separable, there is a pairwise disjoint countable collection of clopen subsets $\mathscr{C}$ of $X$ such that $\bigcup \mathscr{C}$ is dense in $X \backslash A$. Put $F=X \backslash \bigcup \mathscr{C}$. Since $X$ is the inverse limit of the sequence $\left\{X_{\alpha}: \alpha<\omega_{1}\right\}$, there exists $\alpha<\omega_{1}$ and a pairwise disjoint countable collection of clopen subsets $\mathscr{B}$ of $X_{\alpha}$ such that $\left(p_{\alpha}^{\omega_{1}}\right)^{-1}(\bigcup \mathscr{B})=\bigcup \mathscr{C}$. Hence $E=X_{\alpha} \backslash \bigcup \mathscr{B}$ has the property that $\left(p_{\alpha}^{\omega_{1}}\right)^{-1}(E)=F$; hence $E$ is nowhere dense. Pick $\xi<\omega_{1}$ such that $E=E_{\xi}^{\alpha}$ and $\eta \geq \alpha$ such that $g(\eta)=\langle\alpha, \xi\rangle$. By $(\mathrm{G})$ we have that $G=\left(p_{0}^{\eta+1}\right)^{-1}\left(F_{\xi}^{\alpha}\right)$ is contained in $D_{\eta+1}$, and hence by $(\mathrm{C}), p_{\eta+1}^{\omega_{1}} \uparrow F: F \rightarrow G$ is a homeomorphism. Thus, $\beta=\eta+1$ does the job, and $X$ is almost Luzin.

Therefore, $X$ is hereditarily Lindelöf. That $X$ is hereditarily separable as well follows from the following results.

Lemma 4.3. A first-countable, separable almost Luzin space $X$ is hereditarily separable.

Proof. Let $A \subseteq X$, and let $U$ be the (possibly empty) interior of $\bar{A}$. Then $\bar{A} \backslash U$ is nowhere dense and hence of countable weight. This shows that $A \backslash U$ is separable. However, $U \cap A$ is dense in $U$, and $U$ is separable and first-countable. But any dense subset of a separable first-countabe space is separable, so we are done.

Corollary 4.4. A separable compact almost Luzin space is hereditarily separable.

It remains to check that $X$ is $\mathrm{CDH}$. Let $A$ be a countable dense subset of $X$. There is by Lemma 4.2 an index $\alpha<\omega_{1}$ such that $p_{\alpha}^{\omega_{1}}(A) \subseteq D_{\alpha}$ (observe that this implies that $p_{\alpha}^{\omega_{1}}\left\lceil A: A \rightarrow p_{\alpha}^{\omega_{1}}(A)\right.$ is a homeomorphism). So $p_{\alpha}^{\omega_{1}}(A)$ is of the form $E_{\xi}^{\alpha}$. Pick $\beta$ so that $g(\beta)=\langle\alpha, \xi\rangle$. Then $\beta \geq \alpha$ by construction. Hence by $(\mathrm{C})$ and $(\mathrm{F})$, there is a homeomorphism $h: X \rightarrow X$ such that $h(A)=\left(\pi_{0}^{\omega_{1}}\right)^{-1}\left(D_{0}\right)$. From this it is immediate that $X$ is $\mathrm{CDH}$.

Question 4.5. Let $X$ be a compact space such that $X \times X$ is CDH. Does it follow under $\mathrm{CH}$ that $X$ has countable weight?

Let us repeat that Steprāns and Zhou [15] proved that the Cantor cube $2^{\omega_{1}}$ is $\mathrm{CDH}$ under $\mathrm{MA}+\neg \mathrm{CH}$.

\section{ACKNOWLEDGEMENT}

We are indebted to the referee for finding an inaccuracy in one of our arguments.

\section{REFERENCES}

[1] A. V. Arhangel'skii and J. van Mill, Topological homogeneity, 2011, preprint, available online: http://www.few.vu.nl/ vanmill/papers/preprints/preprints.html. To appear in Recent Progress in Topology III.

[2] R. Bennett, Countable dense homogeneous spaces, Fund. Math. 74 (1972), 189-194. $\operatorname{MR} 0301711(46: 866)$ 
[3] C. Bessaga and A. Pełczyński, The estimated extension theorem, homogeneous collections and skeletons, and their applications to the topological classification of linear metric spaces and convex sets, Fund. Math. 69 (1970), 153-190. MR0273347 (42:8227)

[4] L. E. J. Brouwer, On the structure of perfect sets of points, Proc. Akad. Amsterdam 12 (1910), 785-794.

[5] G. Cantor, Beiträge zur Begrundüng der transfiniten Mengenlehre, Math. Ann. 46 (1895), 481-512.

[6] E. K. van Douwen, Retracts of the Sorgenfrey line, Compositio Math. 38 (1979), 155-161. MR.528838(80i:54042)

[7] E. K. van Douwen, The integers and topology, Handbook of Set-Theoretic Topology (K. Kunen and J. E. Vaughan, eds.), North-Holland, Amsterdam, 1984, pp. 111-167. MR776619(85k:54001)

[8] I. Farah, M. Hrušák, and C. Martínez Ranero, A countable dense homogeneous set of reals of size $\aleph_{1}$, Fund. Math. 186 (2005), 71-77. MR2163103 (2006f:54031)

[9] B. Fitzpatrick, Jr. and N. F. Lauer, Densely homogeneous spaces. I, Houston J. Math. 13 (1987), 19-25. MR884229 (88d:54041)

[10] M. K. Fort, Jr., Homogeneity of infinite products of manifolds with boundary, Pacific J. Math. 12 (1962), 879-884. MR0145499 (26:3030)

[11] I. Juhász, Cardinal functions in topology, Mathematical Centre Tracts, vol. 34, Mathematical Centre, Amsterdam, 1971. MR0340021 (49:4778)

[12] K. Kunen, A compact L-space under CH, Topology Appl. 12 (1981), 283-287. MR623736 (82h:54065)

[13] J. R. Porter and R. G. Woods, Extensions and absolutes of Hausdorff spaces, Springer-Verlag, New York, 1988. MR918341 (89b:54003)

[14] W. Sierpiński, Sur une propriété topologique des ensembles dénombrables denses en soi, Fund. Math. 1 (1920), 11-16.

[15] J. Steprāns and H. X. Zhou, Some results on CDH spaces. I, Topology Appl. 28 (1988), 147-154. MR932979 (89c:54070)

Department of Mathematics, Ohio University, Athens, Ohio 45701

E-mail address: arhangel.alex@gmail.com

Department of Mathematics, Faculty of Sciences, Vu University Amsterdam, De BoelelaAn $1081^{a}, 1081$ HV Amsterdam, The Netherlands

E-mail address: j.van.mill@vu.nl 